\title{
Swiprosin-1 deficiency impairs macrophage immune response of septic mice
}

\author{
Su Zhang, ${ }^{1}$ Ye Tu, ${ }^{2}$ Yi-Ming Sun, ${ }^{1}$ Ya Li, ${ }^{1}$ Rong-Mei Wang, ${ }^{1}$ Yongbing Cao, ${ }^{3}$ Ling Li, ${ }^{3}$ Li-Chao Zhang, ${ }^{4}$ \\ and Zhi-Bin Wang ${ }^{1}$ \\ ${ }^{1}$ College of Pharmacy, Second Military Medical University, ${ }^{2}$ Department of Medicine, Shanghai East Hospital, Tongji \\ University, ${ }^{3}$ Shanghai Institute of Vascular Disease, Shanghai University of Traditional Chinese Medicine, and ${ }^{4}$ Department \\ of Pharmacy, Shanghai Municipal Hospital of Traditional Chinese Medicine, Shanghai, China.
}

Despite the fact that many therapeutic strategies have been adopted to delay the development of sepsis, sepsis remains one of the leading causes of death in noncoronary intensive care units. Recently, sepsis-3 was defined as life-threatening organ dysfunction due to a dysregulated host response to infection. Here, we report that swiprosin-1 (also known as EFhd2) plays an important role in the macrophage immune response to LPS-induced or cecal ligation and puncture-induced (CLP-induced) sepsis in mice. Swiprosin-1 depletion causes higher mortality, more severe organ dysfunction, restrained macrophage recruitment in the lung and kidney, and attenuated inflammatory cytokine production (including IL-1 1 , IL-6, TNF- $\alpha$, IL-10, and IFN- $\gamma$ ). The immunosuppression caused by swiprosin-1 deficiency is manifested by impaired bactericidal capacity and decreased HLA-DR expression in macrophages. Swiprosin-1 affects the activation of the JAK2/STAT1/STAT3 pathway by regulating the expression of IFN- $\gamma$ receptors in macrophages. Our findings provide a potential target for the regulation of the macrophage immune response in sepsis.

Authorship note: SZ and YT contributed equally to this work.

Conflict of interest: The authors have declared that no conflict of interests exists.

Submitted: August 9, 2017 Accepted: December 28, 2017 Published: February 8, 2018

Reference information: JCl Insight. 2018;3(3):e95396. https:// doi.org/10.1172/ji.insight.95396.

\section{Introduction}

Sepsis, an uncontrolled host inflammatory response to infection with the presence of organ dysfunction, is still the main cause of death in noncoronary intensive care units, with a mortality rate as high as $30 \%-50 \%$ (1). Although the specific molecular pattern of sepsis is unclear, it is widely accepted that dysregulation of the host response and consecutive defects in homeostasis are critical in the progress of sepsis (2). The concept of hyperinflammatory syndrome, which has dominated septic pathology over the past 2 decades, is currently challenged (3). Clinical trials have demonstrated that the therapeutic outcome of using substances to inhibit inflammation is disappointing (4). Recently, a new definition for sepsis and septic shock named sepsis-3 has eliminated the concept of systemic inflammatory response syndrome (SIRS) (1). On the other hand, the incidence of sepsis is increasing in the aging population that have impaired immunity due to immunosenescence (5). Indeed, most septic patients manifest significant immunosuppression or immunoparalysis, as represented by an impaired activation of immune responses and hypoinflammation (6), which may contribute directly or indirectly to the inability of individuals to ward off the lethal effects of septic challenge (7).

Of all the sepsis-responsive cells, macrophages play a critical role in promotion of the innate immune response and initiation of the adaptive immune response (8), and depletion of macrophages in septic mice increases mortality (9). However, macrophages isolated from a late-phase septic individual exhibit an immunosuppressive phenotype (10). Typically, phagocytic impairment within the innate immune response of macrophages is an important contributor to septic dysfunction $(11,12)$. Moreover, $\mathrm{T}$ cells, which are crucial regulators of macrophage function, are profoundly lost and dysfunctional in sepsis, making the sustained hyperactivation of macrophages difficult (3). Therefore, understanding the mechanisms contributing to immunosuppressive or hyporesponsive characteristics of macrophages during sepsis is important.

Swiprosin-1, also known as EF hand domain-containing protein D2 (EFhd2), was first identified in human lymphocytes, predominantly in $\mathrm{CD}^{+} \mathrm{T}$ lymphocytes (13), and was later identified in immature resting or activated $\mathrm{B}$ cells $(14,15)$ and in nonlymphoid tissues $(16,17)$. The high expression of 
swiprosin-1 in T cells and its participation in BCR-induced apoptosis in immature B cells suggests that swiprosin-1 may be involved in the regulation of the immune response (15). Meanwhile, swiprosin-1 modulates the expression of proinflammatory cytokines (IL-3 and IL-8) in mast cells, and an increased expression of swiprosin-1 is also found in the model tissues of passive cutaneous-anaphylaxis and atopic dermatitis (18). However, the expression and function of swiprosin-1 in macrophages are largely unknown. Here, we found that swiprosin-1 was abundantly expressed in macrophages and responded to LPS challenge. More importantly, we observed that more swiprosin-1-KO mice died of LPS-induced sepsis. Based on these findings, we hypothesized that swiprosin-1 participated in the pathogenesis of sepsis by regulating the immune response of macrophages.

In our study, we used swiprosin-1-deficient mice and an LPS- and CLP-induced septic model to identify the effect of swiprosin-1 in experimental sepsis. Consequently, we assessed systemic and local inflammation, the immune state, tissue injury, macrophage recruitment, and the activation of relative signaling pathways. Finally, we discussed the possible mechanism of swiprosin-1 in regulating the macrophage immune response.

\section{Results}

Swiprosin-1 deficiency leads to increased mortality associated with the phenotypes of immunosuppression in LPS- and CLP-induced sepsis. Mortality increased in swiprosin-1-KO mice treated with an i.p. injection of a lethal dose of LPS (40 mg/kg) compared with that in WT mice (Figure 1A). Although there was no significant difference in the serum levels of IL-1 $\beta$, IL- 6 , and TNF- $\alpha$ between the KO and WT mice, the concentrations of these proinflammatory factors were significantly decreased in the KO mice compared with the WT mice after i.p. LPS (Figure 1B). Meanwhile, the higher septic lethality and mild proinflammation response of the KO mice were also verified in another clinically relevant sepsis model induced by CLP (Figure 1, C and D). Given that IL-10 can negatively regulate proinflammatory factors upon inflammatory stimulation (19), we next examined the production of IL-10 in the serum of the KO mice. Unexpectedly, IL-10 levels were also significantly decreased in the KO mice versus WT mice after i.p. LPS (Figure 1E).

Knowing that macrophages are critical drivers of the innate immune response during infection and inflammation, we used clodronate liposomes (CLOPs) to deplete macrophages in C57BL/6J mice. Twenty-four hours after an i.v. injection of CLOPs $(0.1 \mathrm{ml} / 10 \mathrm{~g}), 87 \%$ of the splenic macrophages were depleted, which confirmed the efficiency of macrophage deprivation (Supplemental Figure 1A; supplemental material available online with this article; https://doi.org/10.1172/jci.insight.95396DS1). To examine the importance of macrophages in LPS-induced sepsis, $3 \times 10^{7}$ peritoneal macrophages were returned to the macrophage-depleted mice before the i.p. LPS treatment. Compared with the $75 \%$ survival rate of the control mice, the macrophage-deprived mice all died within 30 hours, whereas $25 \%$ of the macrophage-complement mice survived at 4 days (Supplemental Figure 1B). Subsequently, the macrophages isolated from KO and WT mice were returned to macrophage-deprived mice. Although all the mice in these 2 groups died, the survival time in the KO group was significantly shorter than that in the WT group (Figure 1F).

As impaired bacterial clearance is a significant manifestation of sepsis-induced immune dysfunction, we assessed whether swiprosin-1 deletion affected the bactericidal ability of septic mice. More bacterial burden was observed in the blood of KO mice than in that of WT mice after i.p. LPS (Figure 1G). Similarly, more bacterial burden was also found in the blood and peritoneal lavage fluid in KO mice than in those of WT mice after CLP (Figure 1H). These data suggest that swiprosin-1 deletion-increased septic death may be due to immune deficiency rather than an inflammatory factor storm.

Swiprosin-1 deficiency aggravates lung and kidney injuries in septic mice. Since sepsis-induced death is associated with severe organ dysfunction, we subsequently examined the pathological changes in the lungs and kidneys of septic mice. After LPS stimulation, inflammatory cell infiltration, edema, hemorrhage, and alveolar septal thickening were observed, while inflammatory cell infiltration and hemorrhage were more severe in $\mathrm{KO}$ mice (Figure 2, A and B). The lung wet/dry (W/D) weight ratio in the KO mice was higher than that in the WT mice after i.p. LPS, which also demonstrated that pulmonary edema was more severe in the KO mice (Figure 2C). Similarly, more severe lung lesions were also observed in the KO mice after CLP (Figure 2, D-F). Unexpectedly, the levels of IL-1 $\beta$, IL-6, and TNF- $\alpha$ in the bronchoalveolar lavage fluid (BALF) were significantly decreased in the KO mice compared with the WT mice after i.p. LPS (Figure 2G). 


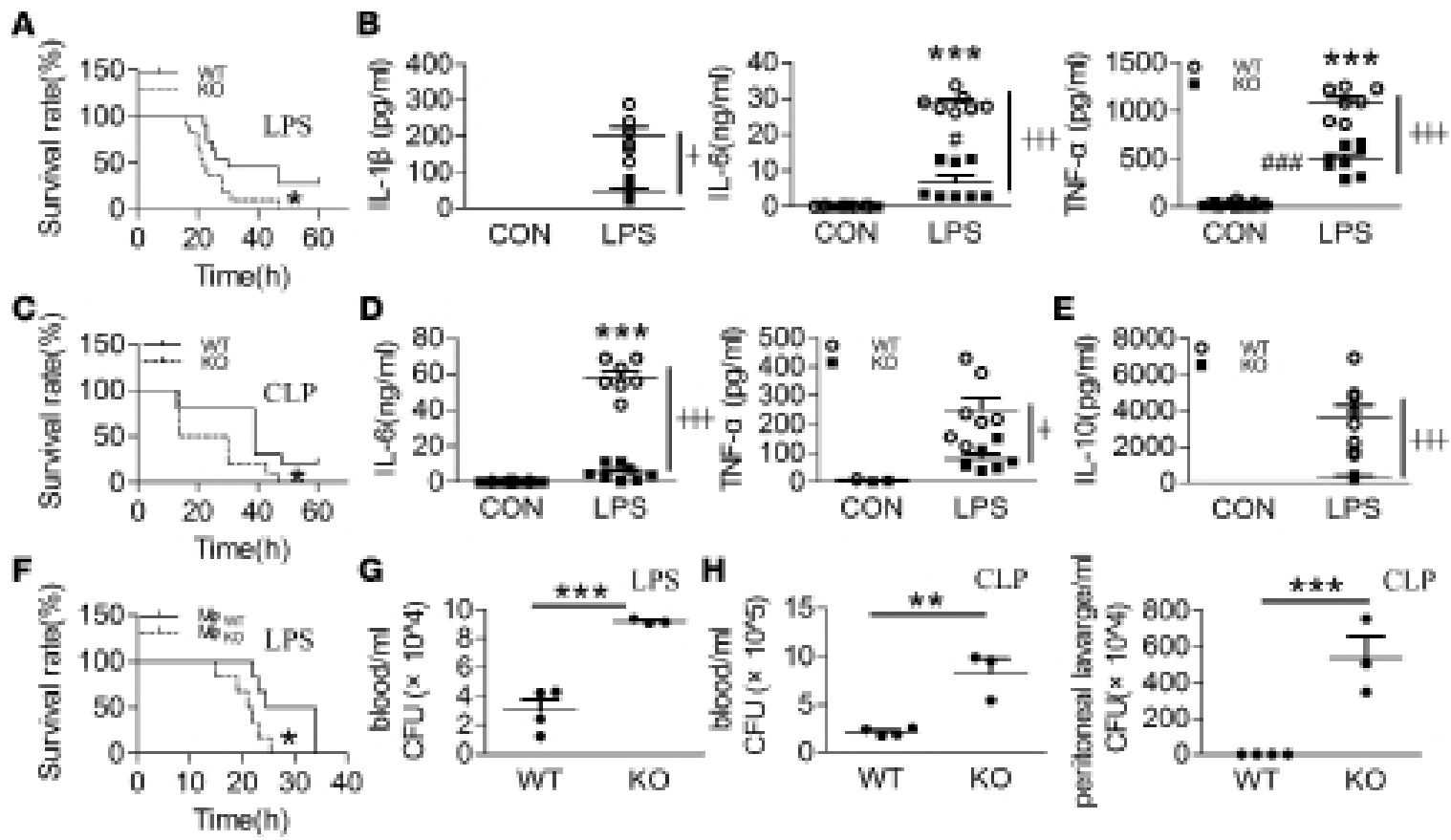

Figure 1. Swiprosin-1-KO mice exhibited higher mortality and immunosuppression after LPS- and CLP-induced sepsis. (A) Survival curves after i.p. LPS (40 mg/kg) $\left(n=11,{ }^{*} P<0.05\right.$, WT vs. KO), Log-rank (Mantel-Cox). (B) The levels of IL-1 $\beta$, IL-6, and TNF- $\alpha$ in the serum after i.p. LPS for 6 hours ( $n=$ 6-8, ${ }^{* * *} P<0.001$, WT control (WT-CON) vs. WT-LPS; ${ }^{*} P<0.05, \# \# P<0.001$, KO-CON vs. KO-LPS; $+P<0.05, H P<0.001$, WT-LPS vs. KO-LPS), 1-way ANOVA (LSD test) ; the results are depicted as the mean \pm SEM. (C) Survival curves after cecal ligation and puncture (CLP) treatment $\left(n=10,{ }^{*} P<0.05\right.$, WT vs. KO), Log-rank (Mantel-Cox). (D) The levels of IL-6 and TNF- $\alpha$ in the serum after CLP treatment for 18 hours ( $n=7$ or 8 , *** $P<0.001$, WT-Sham vs. WT-CLP; ${ }^{+} P<0.05,+H P<0.001$, WT-CLP vs. KO-CLP), 1-way ANOVA (LSD test); the results are depicted as the mean \pm SEM. (E) IL-10 concentration in the serum after i.p. LPS for 6 hours ( $n=8, H P<0.05$, WT-LPS vs. KO-LPS), 1-way ANOVA (LSD test); the results are depicted as the mean \pm SEM. (F) Survival curves after supplementing with WT and KO macrophages in the macrophage-depleted mice after i.p. LPS $\left(n=6,{ }^{*} P<0.05\right.$, WT-M $\varphi$ vs. KO-M $\varphi$ ), Log-rank (Mantel-Cox). (C) Bacterial burden in the blood of the LPS-induced septic mice $\left(n=3,{ }^{* * *} P<0.001\right.$, WT vs. KO), 2-tailed $t$ test; the results are depicted as the mean \pm SEM. (H) Bacterial burden in the blood and peritoneal lavage fluid of the CLP-induced septic mice $\left(n=3,{ }^{* *} P<0.01\right.$, ${ }^{* * *} P<0.001$, WT vs. KO), 2 -tailed $t$ test; the results are depicted as the mean \pm SEM.

Subsequently, a kidney histological examination showed that the septic mice demonstrated excessive inflammatory cell infiltration, RBC trapping, glomerular congestion, and loss of proximal tubular brush border after i.p. LPS. Furthermore, glomerular congestion was more severe in KO mice than in WT mice (Figure 3, A and B). Moreover, the blood urea nitrogen (BUN) and creatinine (Cr) levels in the $\mathrm{KO}$ mice were higher than those in the WT mice after i.p. LPS, which demonstrated that the KO mice exhibited more severe renal damage (Figure 3C). Similarly, the kidney lesions were more severe (Figure 3, D and E) and the $\mathrm{Cr}$ level was higher in the KO mice after CLP (Figure 3F). Conversely, the concentrations of IL-1 $\beta$, IL- 6 , and TNF- $\alpha$ in the kidney homogenates were significantly decreased in the KO mice compared with the WT mice after i.p. LPS (Figure 3G).

Swiprosin-1 deficiency restrains macrophage recruitment and inflammatory factor production. Monocyte/macrophage infiltration is one of the most important factors leading to excessive inflammation. Next, we assessed whether swiprosin-1 affected macrophage recruitment in the target tissues of septic mice. There was less $\mathrm{F} 4 / 80^{+}$macrophage recruitment in the lungs and kidneys of the KO mice after i.p. LPS (Figure $4 \mathrm{~A}$ ) or CLP (Supplemental Figure 2). A decreased percentage of $\mathrm{F} 4 / 80^{+} \mathrm{CD} 11 \mathrm{~b}^{+}$macrophages in the peritoneal lavage fluid or spleen was also observed in the $\mathrm{KO}$ mice after i.p. LPS (Figure 4B). Further, we confirmed that the restrained macrophage recruitment was due to inhibited cell migration rather than impaired cell viability (Supplemental Figure 3).

Knowing that macrophages are an important source of inflammatory factors, we assessed whether swiprosin-1 affected cytokine production in vitro. The mRNA and protein expressions of swiprosin- 1 in the macrophages were increased in a time-dependent manner after LPS treatment (Figure 4C). Subsequently, the mRNA (Supplemental Figure 4) and protein (Figure 4D) levels of IL-1 $\beta$, IL-6, and TNF- $\alpha$ were significantly decreased in the supernatant of peritoneal macrophages isolated from the KO mice after 
A
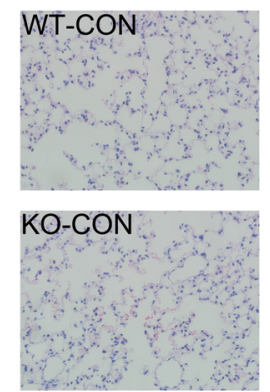

D
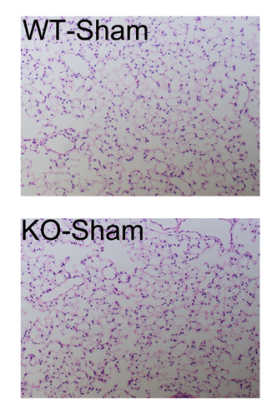

G

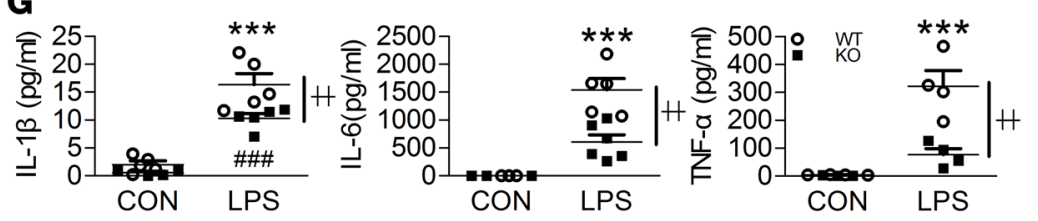

Figure 2. Swiprosin-1 deficiency aggravates the lung injuries in LPS- and CLP-induced sepsis. (A and B) H\&Estained sections and injury scores of the lung after i.p. LPS for 6 hours $\left(n=6\right.$, scale bar: $200 \mu \mathrm{m}$, ${ }^{* *} P<0.001$, WT-CON vs. WT-LPS; \#\#P<0.001, KO-CON vs. KO-LPS; $H P<0.001$, WT-LPS vs. KO-LPS), 1-way ANOVA (LSD test); the results are depicted as the mean $\pm S E M$. (C) Lung W/D ratio after i.p. LPS for 6 hours $\left(n=4,{ }^{*} P<0.05\right.$, WT-CON vs. WT-LPS; ${ }^{\# \# P}<0.001$, KO-CON vs. KO-LPS; $+P<0.05$, $H P<0.01$, WT-LPS vs. KO-LPS), 1-way ANOVA (LSD test); the results are depicted as the mean \pm SEM. (D and $\mathbf{E}$ ) $\mathrm{H} \& \mathrm{E}$-stained sections and injury scores of the lungs after CLP treatment for 18 hours $\left(n=6\right.$, scale bar: $200 \mu \mathrm{m}$, ${ }^{* *} P$ $<0.001$, WT-Sham vs. WT-CLP; \#\#\#<0.001, KO-Sham vs. KO-CLP; HP $<0.001$, WT-CLP vs. KO-CLP), 1-way ANOVA (LSD test); the results are depicted as the mean \pm SEM. (F) Lung W/D ratio after CLP treatment for 18 hours $(n=4-5$, ${ }^{\#} P<0.05$, KO-Sham vs. KO-CLP; $\# P<0.01$, WT-CLP vs. KO-CLP), 1-way ANOVA (LSD test); the results are depicted as the mean \pm SEM. (C) The levels of IL- $1 \beta$, IL-6, and TNF- $\alpha$ in the bronchoalveolar lavage fluid (BALF) after i.p. LPS for 6 hours $\left(n=4-6,{ }^{* * *} P<0.001\right.$, WT-CON vs. WT-LPS; ${ }^{\# \# P} P<$ 0.001 , KO-CON vs. KO-LPS; HP<0.01, WT-LPS vs. KO-LPS), 1 -way ANOVA (LSD test); the results are depicted as the mean \pm SEM.

LPS treatment for 6, 12, and 24 hours. IL-10 levels also significantly decreased in the supernatant of peritoneal macrophages isolated from the KO mice after LPS treatment (Supplemental Figure 5), which was consistent with the result in vivo, indicating that the decreased production of proinflammatory factors was not due to IL-10. Hence, we postulated that swiprosin-1 per se participated in the expression of proinflammatory cytokines. Stable clones with swiprosin-1 overexpression (OE) and GFP control RAW264.7 cells were treated with LPS. The concentrations of IL-1 $\beta$, IL- 6 , and TNF- $\alpha$ were significantly increased in the swiprosin-1 OE RAW264.7 cells (Figure 4E). These results indicated that swiprosin-1 plays a critical role in modulating proinflammatory cytokine production in macrophages.

Swiprosin-1 deficiency inhibits the IFN- $\gamma / J A K 2 / S T A T 1 / S T A T 3$ pathway in LPS-stimulated macrophages. $\mathrm{NF}-\mathrm{\kappa B}, \mathrm{MAPKs}$, and JAK/STAT are recognized as the 3 main inflammatory signaling pathways in macrophages, and the activation of these pathways plays a key role in regulating the production of proinflammatory mediators (20). Here, we found that the NF- $\mathrm{B}$ (phosphorylation of $\mathrm{I} \kappa \mathrm{B} \alpha, \mathrm{IKK} \alpha / \beta$, and p65) and MAPKs (phosphorylation of $\mathrm{p} 38, \mathrm{JNK}$, and ERK) pathways were both activated regardless of the presence or absence of swiprosin-1 (Supplemental Figure 6). However, the activation of the JAK/STAT pathway (phosphorylation of JAK2, STAT1, and STAT3) was significantly decreased in the swiprosin-1 KO macrophages compared with the WT macrophages after LPS treatment (Figure 5A). In contrast, OE of swiprosin-1 remarkably augmented the phosphorylation of JAK2, STAT1, and STAT3 (Figure 5B). Since IFN- $\gamma$ is an important activator of the JAK/STAT pathway, we further examined IFN- $\gamma$ production in septic mice. Compared with the WT mice, there was less IFN- $\gamma$ production in the serum of the KO mice after LPS treatment (Figure 5C). Although macrophages are not the main cells releasing IFN- $\gamma$, they can produce autocrine IFN- $\gamma$. We therefore detected the level of IFN- $\gamma$ produced by macrophages. Similarly, IFN- $\gamma$ production and gene expression were significantly decreased in the KO macrophages after the LPS treatment (Supplemental Figure 7A). Moreover, given that IFN- $\gamma$ is mainly released by T cells, we also observed IFN- $\gamma$ production in the $\mathrm{T}$ cells isolated from the $\mathrm{KO}$ mice. The IFN- $\gamma$ concentration was also significantly lower in the KO T cells after LPS treatment (Supplemental Figure 7B). In contrast, swiprosin-1 had no effect on the mRNA expression of LPS pattern recognition receptors TLR4/CD14 (Supplemental Figure 8). 
A

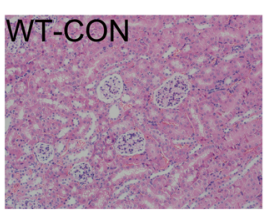

B

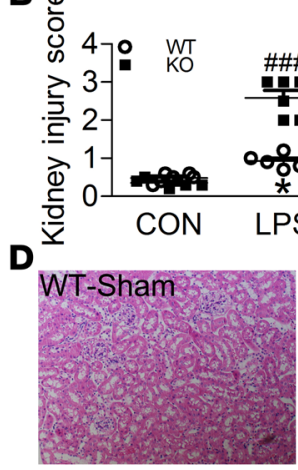

E 은

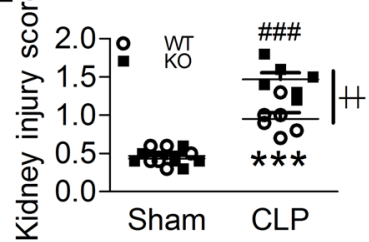

G

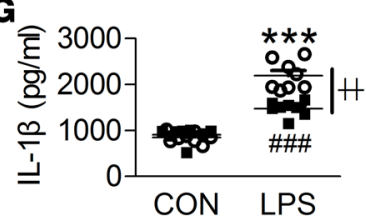

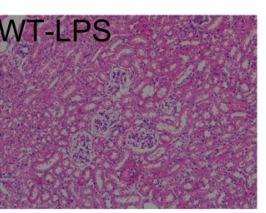
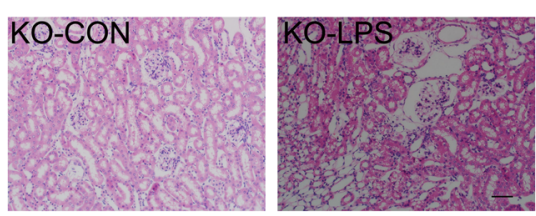

C
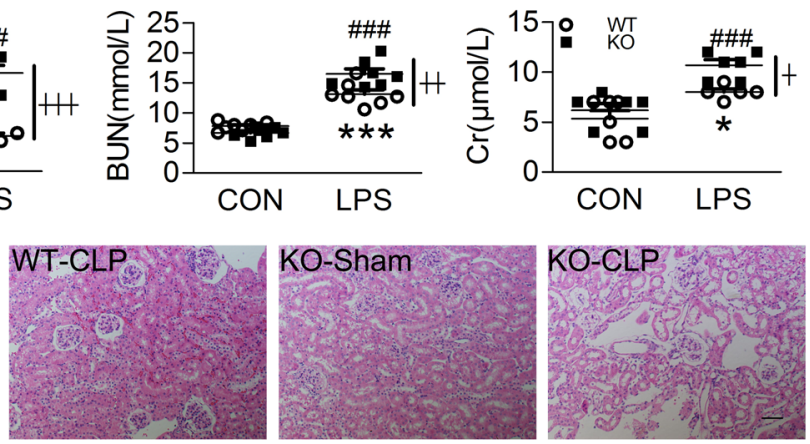

$\mathbf{F}$
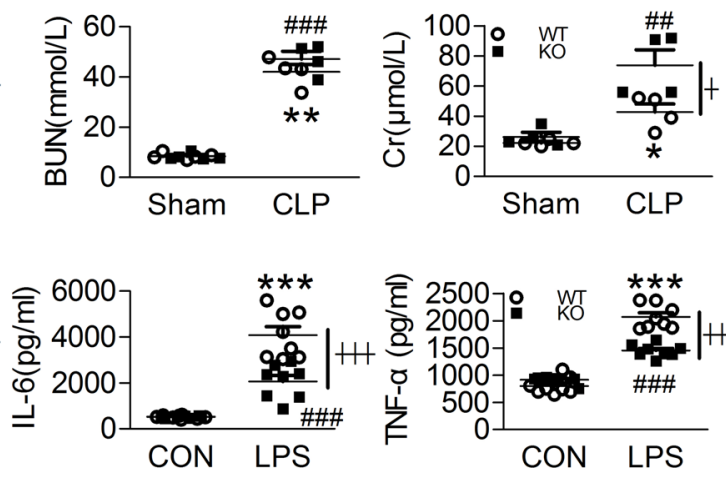

Figure 3. Swiprosin-1 deletion aggravates kidney injuries in LPS- and CLP-induced sepsis. ( $A$ and B) H\&E-stained sections and injury scores of the kidney after i.p. LPS for 6 hours ( $n=6$, scale bar: $200 \mu \mathrm{m},{ }^{*} P<0.05$, WT-CON vs. WT-LPS; ${ }^{\# \# \#<<}$ $0.001, \mathrm{KO}-\mathrm{CON}$ vs. KO-LPS; HP<0.001, WT-LPS vs. KO-LPS), 1-way ANOVA (LSD test); the results are depicted as the mean \pm SEM. (C) Concentrations of the blood urea nitrogen (BUN) and creatinine (Cr) after i.p. LPS for 6 hours $\left(n=5-7,{ }^{*} P<0.05\right.$,

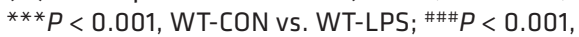
KO-CON vs. KO-LPS; ${ }^{+} P<0.05, H_{P}<0.01$, WT-LPS vs. KO-LPS), 1-way ANOVA (LSD test); the results are depicted as the mean \pm SEM. ( $\mathbf{D}$ and $\mathbf{E}$ ) H\&Estained sections and injury scores of the kidneys after CLP treatment for 18 hours $(n=6$, scale bar: $200 \mu \mathrm{m},{ }^{* *} P<0.05$, WT-Sham vs. WT-CLP; ${ }^{* \#} P$ $<0.001$, KO-Sham vs. KO-CLP; $H P<0.01$, WT-CLP vs. KO-CLP), 1-way ANOVA (LSD test); the results are depicted as the mean \pm SEM. (F) Concentrations of the blood urea nitrogen (BUN) and creatinine (Cr) after CLP treatment for 18 hours $\left(n=4-5,{ }^{*} P<\right.$ 0.05 , WT-Sham vs. WT-CLP; ${ }^{*} P<0.01$, WT-Sham vs. WT-CLP; \#\# $<0.01$, KO-Sham vs. KO-CLP; \#\#P $<0.001 \mathrm{KO}-$ Sham vs. KO-CLP; $+P<0.05$, WT-CLP vs. KO-CLP), 1-way ANOVA (LSD test); the results are depicted as the mean \pm SEM. (C) IL-1 $\beta$, IL-6, and TNF- $\alpha$ concentrations of the kidney homogenates after i.p. LPS for 6 hours $\left(n=8,{ }^{* *} P<\right.$ 0.001 , WT-CON vs. WT-LPS; ${ }^{\# \# P<0.001, ~ K O-C O N ~}$ vs. KO-LPS; $H P<0.01, H+P<0.001$, WT-LPS vs. KO-LPS), 1-way ANOVA (LSD test); the results are depicted as the mean \pm SEM.

Considering that the lack of IFN- $\gamma$ might be the cause of the insufficient activation of the JAK/ STAT pathway, we investigated whether the addition of IFN- $\gamma$ could augment proinflammatory cascades in $\mathrm{KO}$ macrophages. Administration of IFN- $\gamma(50 \mathrm{ng} / \mathrm{ml})$ eliminated the decreased expression of IL-1 $\beta$ in KO macrophages compared with WT macrophages with or without LPS (Figure 5D). However, IL-6 levels were still significantly decreased in the KO macrophages under IFN- $\gamma$ stimulation combined with LPS (Figure 5D). Moreover, the TNF- $\alpha$ level was still lower in the KO macrophages after IFN- $\gamma$ treatment with or without LPS (Figure 5D). Then, we assessed the activation of the JAK/STAT pathway after IFN- $\gamma$ treatment. Neither IFN- $\gamma$ alone nor LPS plus IFN- $\gamma$ could eliminate the insufficient activation of JAK2/STAT1/STAT3 due to the absence of swiprosin-1 (Figure 6A). These results suggest that IFN- $\gamma$ could not effectively recover the swiprosin-1 deletion-induced decrease in proinflammatory response in macrophages.

As IFN- $\gamma$ activates the JAK/STAT pathway by binding to the IFN- $\gamma$ receptor (IFN- $\gamma \mathrm{R}$ ), we further examined whether swiprosin-1 deletion affects IFN- $\gamma$ R expression. The results showed that LPS significantly upregulated IFN- $\gamma \mathrm{R} \alpha$ and IFN- $\gamma \mathrm{R} \beta$, while the upregulation of IFN- $\gamma \mathrm{R}$ in $\mathrm{KO}$ macrophages was significantly lower than that in WT macrophages (Figure 6B). Nevertheless, the coimmunoprecipitation result showed that the IFN- $\gamma \mathrm{R} \alpha$ did not directly interact with swiprosin-1 (Figure 6C).

Furthermore, human macrophage THP-1 cell showed an upregulation of swiprosin-1 under LPS treatment (Supplemental Figure 9A). In addition, knockdown of swiprosin-1 in THP-1 cells using siRNA reduced the levels of IL-6 and TNF- $\alpha$ in the supernatant after LPS treatment (Supplemental Figure 9B). Furthermore, the activation of the JAK/STAT pathway was also reduced in siRNA-treated THP-1 cells (Supplemental Figure 9C). These results further confirmed the role of swiprosin-1 in regulating human macrophages' response to LPS.

Swiprosin-1 deficiency impairs the LPS-induced immune response. Sepsis is accompanied by an immunosuppressive response, and IFN- $\gamma$ plays an important role in initiating and modulating macrophage immune 
A

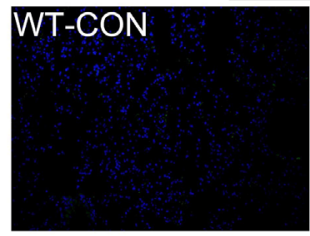

KO-CON

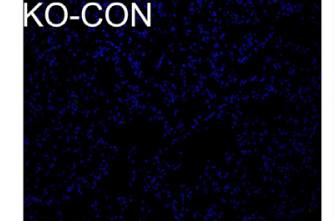

B peritoneal lavage

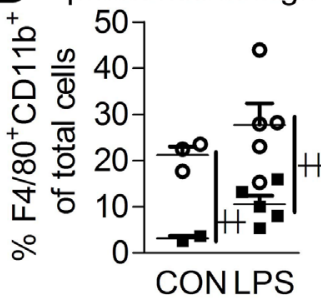

Lung

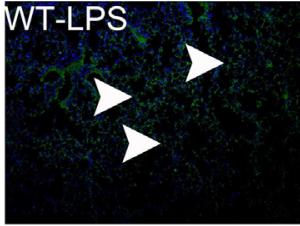

KO-LPS

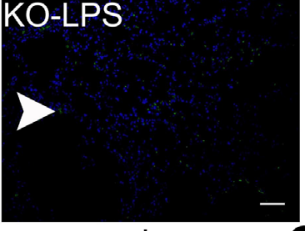

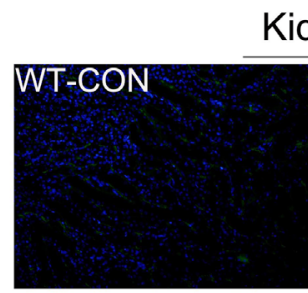

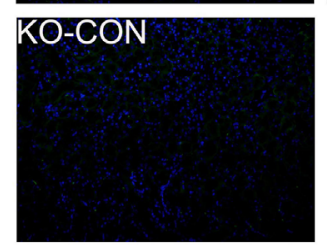

Kidney

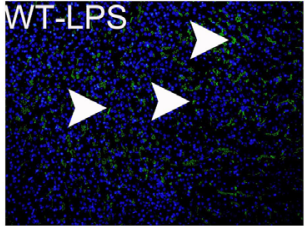

KO-LPS

Time(h) swiprosin-1 $0 \quad 6$

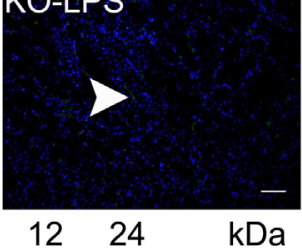
+o $57 \mathrm{~F}^{\mathrm{WT}}$ GAPDH

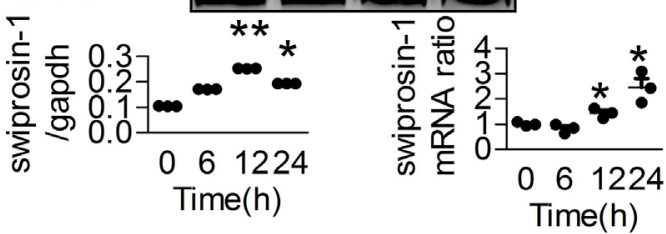

\section{D}

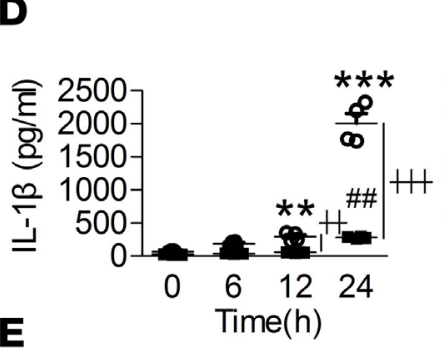

CONLPS

Time(h)

Time(h)

E
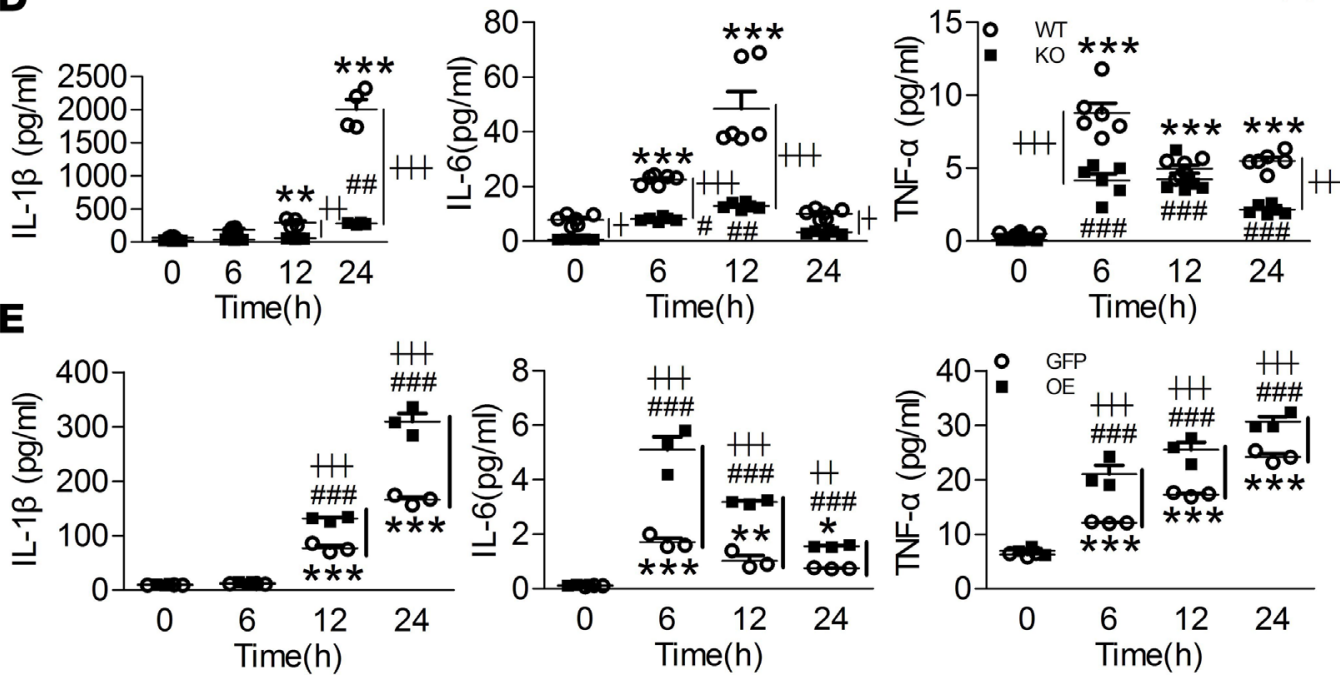

Figure 4. Swiprosin-1 deficiency restrains macrophage recruitment and attenuates proinflammatory cytokine production after LPS treatment. (A) $\mathrm{F} 4 / 80^{+}$cells in the lung and kidney after i.p. LPS for 6 hours (green fluorescence, F4/80+ macrophage; blue fluorescence, nucleus; scale bar: $200 \mu \mathrm{m}$ ). (B) The percentage of $\mathrm{F} 4 / 80^{+} \mathrm{CD} 11 \mathrm{~b}^{+}$macrophages in the peritoneal lavage fluid and spleen after i.p. LPS for 6 hours $(n=3-5$, $\# \#<0.01, \mathrm{KO}-\mathrm{CON}$ vs. KO-LPS; $+P<0.05,+P<0.01$, WT-LPS vs. KO-LPS), 1-way ANOVA (LSD test); the results are depicted as the mean \pm SEM. (C) The expression of swiprosin-1 in the WT macrophages after LPS treatment $\left(n=3,{ }^{* *} P<0.01,{ }^{*} P<0.0512\right.$ hours vs. 0 hour; ${ }^{*} P<0.05,24$ hours vs. 0 hour), 1 -way ANOVA (LSD test); the results are depicted as the mean \pm SEM. (D) IL-1 $\beta$, IL-6, and TNF- $\alpha$ concentrations in the macrophages after LPS treatment $\left(n=4\right.$ or 6 , ${ }^{* *} P<0.01$, ${ }^{* * *} P$ $<0.001$, WT-LPS vs. WT-0 hour; ${ }^{\#} P<0.05$, $\# P<0.01$, ${ }^{\# \# P}<0.001$, KO-LPS vs. KO-0 hour; $+P<0.05, H P<0.01, H P<0.001$, WT-LPS vs. KO-LPS at the same time point), 1-way ANOVA (LSD test); the results are depicted as the mean \pm SEM. (E) IL-1 $\beta$, IL-6, and TNF- $\alpha$ concentrations in the swiprosin-1 overexpression and GFP-control RAW cells after LPS treatment $\left(n=3,{ }^{*} P<0.05,{ }^{* *} P<0.01,{ }^{* * *} P<0.001\right.$, GFP-LPS vs. GFP-0 hour; $\# \# \#<0.001,0$ E-LPS vs. OE-0 hour; $+P>0.01, H P<0.001$, GFP-LPS vs. OE-LPS at the same time point), 1-way ANOVA (LSD test); the results are depicted as the mean \pm SEM.

responses. Hence, we assessed whether swiprosin-1 deletion affects the innate immune response of macrophages. Monocyte HLA-D related (HLA-DR) downmodulation is considered a major factor of immunosuppression (21). Less HLA-DR $\alpha$ expression was observed in KO macrophages than in WT macrophages after LPS treatment (Figure 7A). In addition, the numbers of white blood cells, lymphocytes, and granulocytes and the percentage of monocytes were significantly decreased in KO mice versus WT mice (Figure 7, B-E). Subsequently, impaired phagocytosis and bacteria-killing abilities were also observed in KO macrophages (Figure 7F). Administration of IFN- $\gamma$ partially restored the phagocytosis and bacteria-killing abilities in the KO macrophages (Figure 7G). 
A

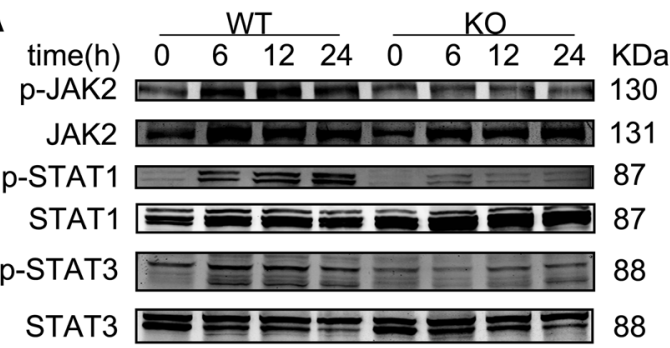

GAPDH 36

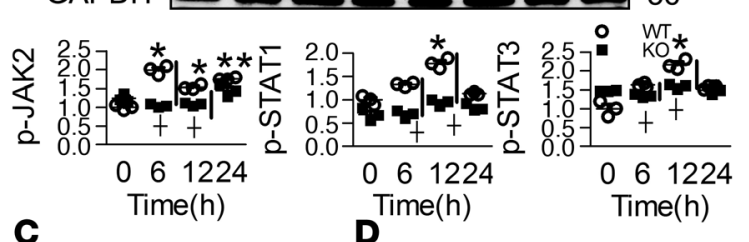

B time(h) $\begin{array}{llllllllll}0 & 6 & 12 & 24 & 0 & 6 & 12 & 24 & \mathrm{kDa}\end{array}$

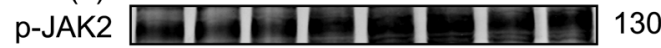

JAK2 EERE IE 130

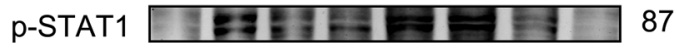

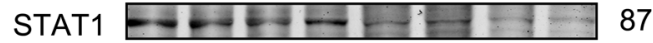

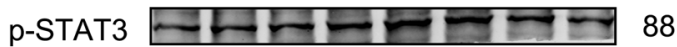

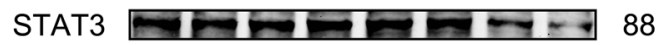

Tubulin एकाष्काष 55

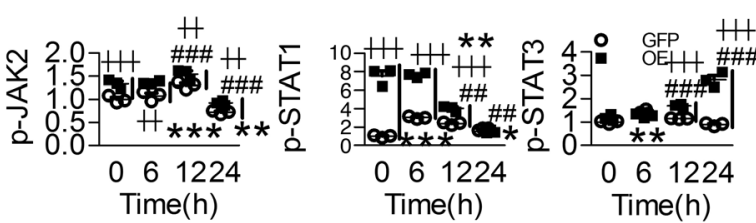

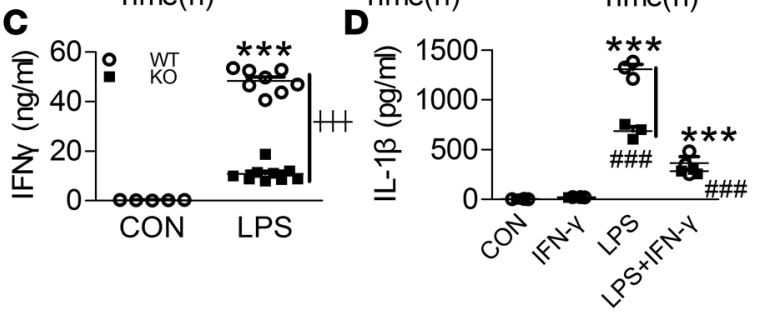

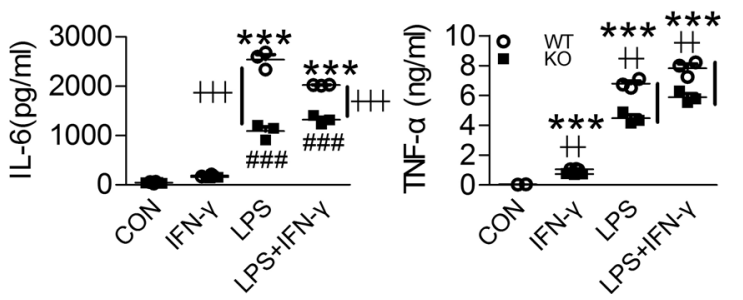

Figure 5. Swiprosin-1 deficiency attenuates the activation of the JAK2/STAT1/STAT3 pathway and the expression of IFN- $\gamma$ R in LPS-treated peritoneal macrophages. (A) The activation of the JAK/STAT pathway in macrophages after LPS treatment $\left(n=3,{ }^{*} P<0.05,{ }^{* *} P<0.01\right.$ WT-LPS vs. WT-0 hour; $+P<$ 0.05 , WT-LPS vs. KO-LPS at the same time point), 1-way ANOVA (LSD test); the results are depicted as the mean \pm SEM. (B) JAK/STAT signaling activation

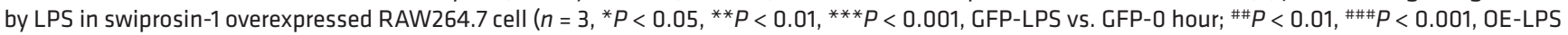
vs. OE-Oh; $+P<0.01, H P<0.001$, GFP-LPS vs. OE-LPS at the same time point), 1-way ANOVA (LSD test); the results are depicted as the mean \pm SEM. (C) Serum level of IFN- $\gamma$ after LPS treatment for 6 hours $\left(n=5-9,{ }^{* * *} P<0.001\right.$, WT-LPS vs. WT-CON; HTP<0.001, WT-LPS vs. KO-LPS), 1-way ANOVA (LSD test); the results are depicted as the mean \pm SEM. (D) IL-1 $\beta$, IL-6, and TNF- $\alpha$ levels in the macrophages after IFN- $\gamma$ treatment for 12 hours ( $n=3$, ${ }^{* *} P<0.01$, ${ }^{* * *} P<0.001$ WT-treatment vs. WT-CON; ${ }^{\# \# P} P 0.001$, KO-treatment vs. KO-CON; $H P<0.01, H_{P}<0.001$, WT vs. KO with same treatment), 1-way ANOVA (LSD test); the results are depicted as the mean \pm SEM.

\section{Discussion}

The pathogenesis of sepsis is complex and poorly understood. Although sepsis is accompanied by an inflammatory storm, the clinical outcomes from many antiinflammatory agents for sepsis therapy indicate that cytokine levels are poorly correlated with mortality (22). Our study showed that swiprosin-1 deficiency increased sepsis-associated mortality and decreased the production of inflammatory cytokines. Interesting

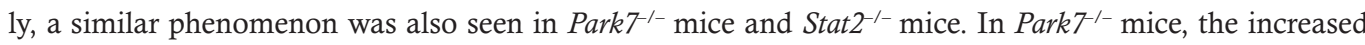
mortality was mainly associated with aggravated lung injury and immunosuppression, along with the impaired bactericidal ability of macrophages (23). Here, we also found that swiprosin-1 deletion caused severe lung and kidney injury and immune paralysis, including impaired bacterial clearance and decreased HLA-DR in the macrophages. Hence, we still observed the expression of Park7 in the macrophages isolated from swiprosin- $-^{-1-}$ mice, whereas no difference was found in the control (Z. Wang, unpublished observations). In Stat $2^{--}$mice, the mortality increased, and the inflammatory response decreased, both of which were associated with hepatitis, peritonitis, and inflammatory cell extravasation (24). We mentioned above that swiprosin-1 deletion affected the phosphorylation of STAT1 and STAT3 activated by type II IFN (IFN- $\gamma$ ). However, STAT2 is an essential transcription factor in type I IFN-mediated antiviral and antiproliferative signaling (25). Therefore, the association between swiprosin-1 and STAT2 was not discussed here.

Swiprosin-1 has been reported to affect cell migration by remodeling the actin cytoskeleton (26-28). Our results showed that swiprosin-1 deletion restrained macrophage recruitment, which partly explained the attenuated proinflammatory cytokine production in the local tissues. In vitro, decreased proinflammatory factors caused by swiprosin-1 deletion reflected the impaired response of macrophage to LPS stimulation. Similarly, Munoz reported that LPS-stimulated macrophages from septic patients had profound decreases in the production of TNF- $\alpha$, IL-1 $\beta$, and IL-6 (29). However, the immune state of swiprosin-1-KO mice was not exactly the same as that in the immunosuppressive phase of septic patients and animals. Here, 
A

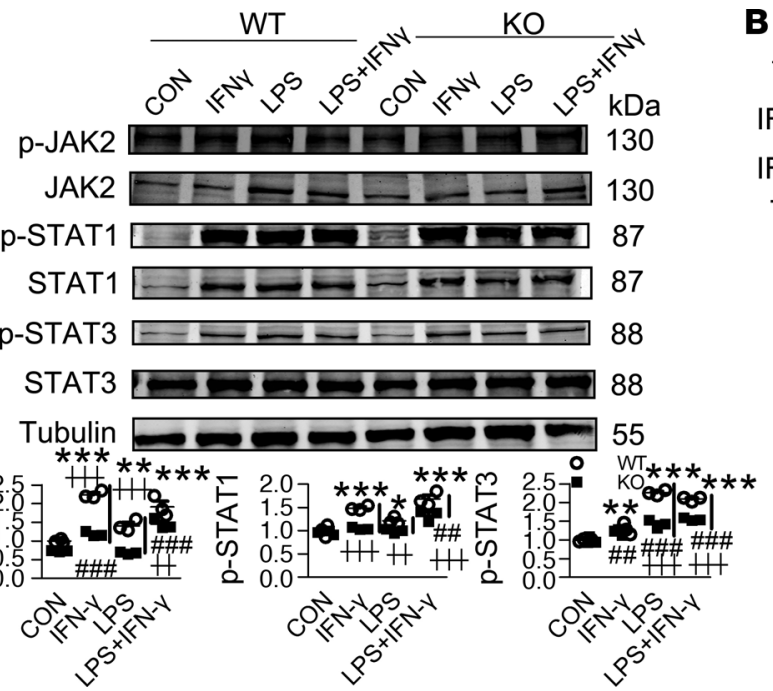

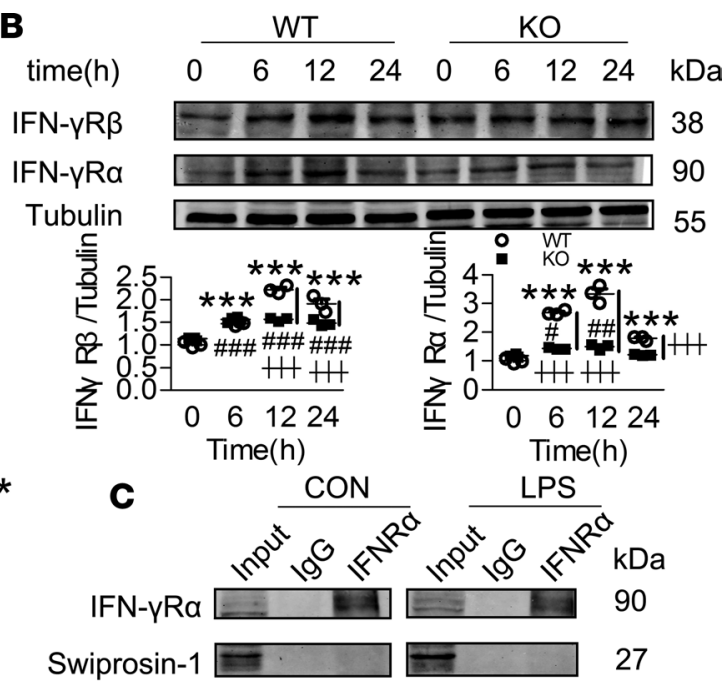

Figure 6. Swiprosin-1 deficiency attenuates the expression of IFN- $\gamma \mathbf{R}$ in LPS-treated peritoneal macrophages. (A) Activation of the JAK/STAT pathway after IFN- $\gamma$ treatment for 12 hours $\left(n=3,{ }^{*} P<0.05\right.$, ${ }^{* *} P<0.01,{ }^{* * *} P<0.001$ WT-treatment vs. WT-CON; \#\# $P<0.01$, \#\#\# $P<0.001$, KO-treatment vs. KO-CON; $+P<0.01, H P<0.001$, WT vs. KO with same treatment), 1-way ANOVA (LSD test); the results are depicted as the mean \pm SEM. (B) The expression of IFN- $\gamma \mathrm{R}$ in macrophages after LPS treatment for 12 hours $\left(n=3,{ }^{* * *} P<0.001\right.$ WT-LPS vs. WT- 0 hour; ${ }^{\#} P<0.05$, ${ }^{\# \#} P<0.01$, \#\#\# $P<0.001$, KO-LPS vs. KO-O hour; $H P<0.001$, WT-LPS vs. KO-LPS at the same time point), 1 -way ANOVA (LSD test); the results are depicted as the mean \pm SEM. (C) Interaction between swiprosin- 1 and IFN- $\gamma$ R $\alpha$ in the macrophages after LPS treatment.

less IL-10 induction was detected in the serum and supernatant of macrophages in the swiprosin-1-deletion conditions. By contrast, the antiinflammatory response in the late stage of sepsis, which yields immunoparalysis, typically involves the production of IL-10 (30). Further, we confirmed that swiprosin-1 directly participated in cytokine production through a JAK/STAT-dependent pathway.

More recently, accumulating evidence indicates that immune paralysis appears at the early stage of sepsis, and this stage seems to be responsible for macrophage activation and inflammatory cytokine production (31). A low expression of HLA-DR in monocytes is an important symbol of impaired monocyte function, including insufficient TNF- $\alpha$ and IL-1 $\beta$ release in response to pathogens $(32,33)$. Furthermore, low monocyte HLA-DR expression is related to an increased risk of nosocomial infections and death $(34,35)$. Here, decreased HLA-DR $\alpha$ expression and impaired bactericidal ability in LPS-stimulated KO macrophages indicated a potential immunosuppression status, which might impair the ability of macrophages against invading pathogens and result in death. IFN- $\gamma$, a potent macrophage activator, has been reported to produce encouraging results in septic patients with decreased monocyte HLA-DR expression and reduced amounts of TNF- $\alpha$ after LPS stimulation (36). In our work, we demonstrated that swiprosin- 1 deletion decreased IFN- $\gamma$ production, which might subsequently impair macrophage activation, suggesting that swiprosin- 1 might act as a potential regulator of the macrophage immune response activated by IFN- $\gamma$. Furthermore, characteristics of immune paralysis were observed in our sepsis model of swiprosin-1-KO mice, suggesting that it might serve as an ideal animal model for studying the immunosuppression of sepsis.

The JAK/STAT signaling pathway regulates numerous processes, such as cell proliferation, differentiation, migration, and survival. IFN- $\gamma$ activates the JAK/STAT signaling pathway by binding to IFN- $\gamma$ R. Our study revealed that swiprosin- 1 deletion attenuated LPS-induced IFN- $\gamma$ R expression. IFN- $\gamma \mathrm{R}$ consists of 2 heterodimeric subunits, IFN- $\gamma \mathrm{R} \alpha$ (ligand binding subunit) and IFN- $\gamma \mathrm{R} \beta$ (signal-transducing subunit). IFN- $\gamma$ R $\alpha$ partly colocalizes with the ganglioside GM1, a marker of lipid-rafts (37). The IFN- $\gamma$ R complex is located within the intact lipid raft microdomain and controls its signal transduction. The depletion or sequestration of cholesterol from the disrupted plasma membrane rafts not only affects the IFN- $\gamma$-inducible phosphorylation of STAT1, but also disturbs transcriptional signaling (38). Swiprosin-1 is identified as a potentially novel lipid raft involved in the detergent-resistant membrane-regulated (DRM-regulated) transduction of BCR signals (14, 39). Swiprosin-1 deletion attenuated IFN- $\gamma$ R expression and inhibited JAK/STAT signaling, which might be correlated with the 
A
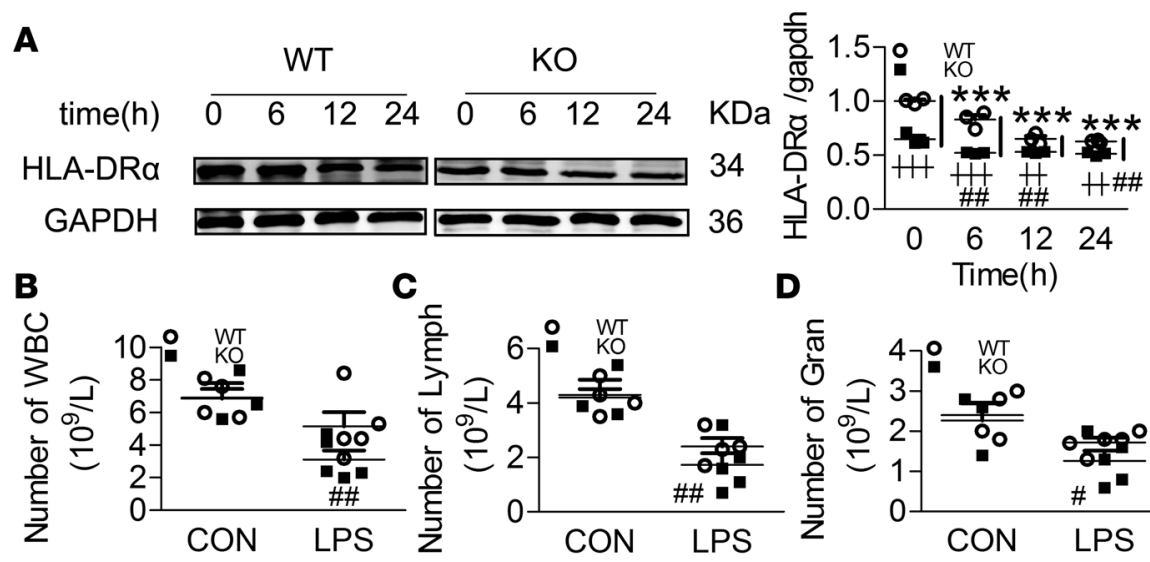

Time(h)
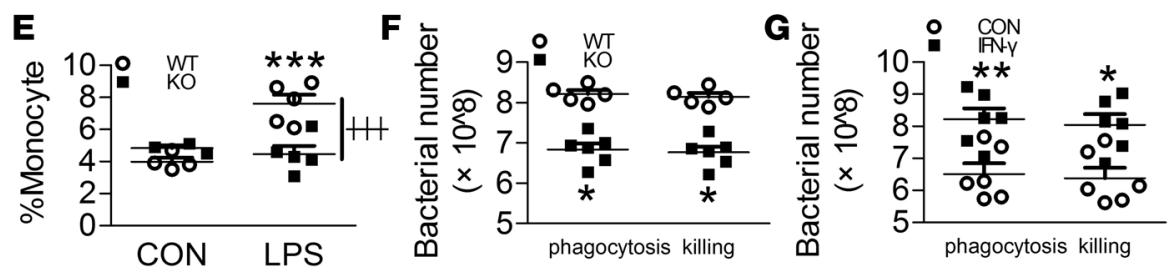

Figure 7. Swiprosin-1 deficiency impairs the LPS-induced immune activation in peritoneal macrophages. (A) The expression of HLA-DR $\alpha$ in macrophages after LPS treatment ( $n=3,{ }^{* *} P<0.001$ WT-LPS vs. WT-O hour; ${ }^{\#} P<0.01$, KO-LPS vs. KO-0 hour; $+P<0.01, H+P<0.001$, WT-LPS vs. KO-LPS at the same time point), 1-way ANOVA (LSD test); the results are depicted as the mean \pm SEM. (B-D) Numbers of white blood cells (WBC), lymphocytes (Lymph), and granulocytes (Gran) in the blood after LPS treatment for 6 hours ( $n=3-5,{ }^{*} P<0.05,{ }^{\#} P<0.01$, KO-LPS vs. KO-CON), 1-way ANOVA (LSD test); the results are depicted as the mean \pm SEM. (E) Percentage of monocytes in the blood after LPS treatment for 6 hours $\left(n=5,{ }^{* *} P<0.001\right.$, WT-LPS vs. WT-CON; H $P<0.001$, WT-LPS vs. KO-LPS), 1-way ANOVA (LSD test); the results are depicted as the mean \pm SEM. (F) Phagocytosis and bacteria-killing ability in the macrophages ( $n=6$, ${ }^{*} P<$ 0.05 , WT vs. KO), 2-tailed $t$ test; the results are depicted as the mean \pm SEM. (G) Phagocytosis and bacteria-killing ability in the swiprosin-1-KO macrophages after IFN- $\gamma(50 \mathrm{ng} / \mathrm{ml})$ treatment $\left(n=6,{ }^{*} P<0.05,{ }^{* *} P<0.01\right.$, IFN- $\gamma$ vs. CON), 2-tailed $t$ test; the results are depicted as the mean \pm SEM.

destruction of the intact lipid raft structure, thus decreasing the amount of IFN- $\gamma \mathrm{R}$ on the cytomembrane and subsequently inhibiting the activation of the JAK/STAT pathway. However, our conjecture remains to be further confirmed.

In summary, our study demonstrated that swiprosin-1 plays an important role in the macrophage immune response to LPS- and CLP-induced sepsis. Swiprosin-1 depletion caused higher mortality and more severe tissue injuries, restrained macrophage recruitment in the lung and kidney, and attenuated inflammatory cytokine production. The immunosuppression caused by swiprosin- 1 deficiency was manifested by decreased bactericidal capacity and reduced HLA-DR expression in the macrophages. In addition, swiprosin-1 affected the activation of JAK2/STAT1/STAT3 pathway by regulating the expression of IFN- $\gamma$ R. We speculate that this may be due to the regulation of lipid rafts by swiprosin-1, which needs further study.

\section{Methods}

Supplemental Methods are available online with this article.

Materials. LPS (E. coli 0111:B4) was obtained from MilliporeSigma. Recombinant murine IFN- $\gamma$ was purchased from PeproTech. PMA (phorbol-12-myristate-13-acetate, S1819) and the GAPDH (mouse mAb, AG019), tubulin (mouse mAb, AT819), p38 (mouse mAb, AM065), phospho-p38 (mouse mAb, AM063), JNK/SAPK (rabbit pAb, AJ518), and phospho-JNK/SAPK (mouse mAb, AJ516) antibodies were all from Beyotime Biotechnology. P44/42 MAPK (Erk1/2) (rabbit mAb, 4695), phospho-p44/42 MAPK (Erk1/2) (rabbit mAb, 4370), IкB $\alpha$ (rabbit mAb, 4812), phospho-IкB $\alpha$ (mouse mAb, 9246), phospho-IKK $\alpha / \beta$ (rabbit mAb, 2697), and phospho-p65 (rabbit mAb, 3033) antibodies were obtained from Cell Signaling Technology. IKK $\alpha / \beta$ (rabbit pAb, sc-7606), P65 (rabbit pAb, sc-372), HLA-DR $\alpha$ (mouse mAb, sc55593), and IFN- $\gamma \mathrm{R} \alpha$ (mouse mAb, sc12755) antibodies were from Santa Cruz Biotechnology Inc. Swiprosin-1/ EFHD2 (goat pAb, ab24368), STAT1 (mouse mAb, ab3987), phospho-STAT1 (mouse mAb, ab29045), STAT3 (mouse mAb, ab119352), phospho-STAT3 (rabbit mAb, ab76315), JAK2 (rabbit mAb, ab108596), phospho-JAK2 (rabbit mAb, ab32101), and IFN- $\gamma \mathrm{R} \beta$ (rabbit pAb, ab77246) antibodies were obtained from 
Abcam. RAW264.7 cells and THP-1 cells were purchased from ATCC. Swiprosin-1-KO mice were constructed by Tong-Hui Ma (Dalian Medical University, Dalian, China) and Wei-Heng Su (Jilin University, Jilin, China). Chemicals were obtained from Sinopharm Chemical Reagent Co.

Animals. Male C57BL/6J mice (18-22 g) were purchased from Shanghai SLAC Laboratory Animal Co. The swiprosin-1-KO mice were generated as described previously (40). All mice were kept under an automated 12-hour dark-light cycle at a controlled temperature of $22^{\circ} \mathrm{C} \pm 2{ }^{\circ} \mathrm{C}$ and a relative humidity of $50 \%$ $60 \%$ with free access to standard dry diet and tap water (Shanghai Puluteng Biological Technology Co.).

Sepsis model. Sepsis was induced by a single i.p. injection of LPS (40 mg/kg) or CLP, as described previously (41). The mice were completely anesthetized with $0.75 \%$ pentobarbital solution $(10 \mu \mathrm{g} / \mathrm{g})$, and a midline abdominal incision was performed. The ileocecal valve was ligated on the distal 3/4 of the cecum. The cecum was perforated with 2 holes by a through-and-through puncture with a 21-gauge needle in a sterile environment, and a droplet of feces was extruded from holes to induce polymicrobial peritonitis. The abdominal wall was sutured in 2 layers and injected s.c. with $1 \mathrm{ml}$ of a $0.9 \%$ sodium chloride solution for fluid resuscitation. The animals in the sham group underwent laparotomy and bowel manipulation without ligation and perforation. All mice had free access to food and water after recovery from anesthesia.

Measurement of bacterial burden. Twelve hours after i.p. injection of LPS ( $40 \mathrm{mg} / \mathrm{kg}$ ) or 18 hours after CLP, the mice were sacrificed to collect blood samples and peritoneal fluid. The diluted specimens were plated onto an LB agar plate and were incubated at $37^{\circ} \mathrm{C}$ for 24 hours. The results are presented as CFU $/ \mathrm{ml}$.

Tissue injury study. Mice were sacrificed 6 hours after i.p. LPS (40 mg/kg) or 18 hours after CLP. The wet weight of the whole lung was measured, and the lung was then desiccated at $65^{\circ} \mathrm{C}$ for 72 hours to determine the dry weight. BUN and Cr were measured on a HITACHI 7600-120 system (HITACHI) according to the manufacturer's instructions. The tissues were cut into $5-\mu \mathrm{m}$ sections and H\&E stained. The histological characteristics of the lung injury - including alveolar edema and hemorrhage, the number of infiltrating leukocytes, and the thickness of the alveolar wall and epithelium — were evaluated. Kidney injury was observed with respect to excessive inflammatory cell infiltration, RBC trapping, glomerular congestion, and loss of the proximal tubular brush border. Each histological characteristic was evaluated on a scale of 0 to 3 ( 0 , normal; 1 , mild; 2 , moderate; 3 , severe).

Generation of stable clones of the swiprosin-1 OE-RAW cell line. Swiprosin-1-OE lentiviruses were produced as described previously (40). Briefly, recombinant lentiviruses expressing the CDS sequence (residues 54-776 in the murine swiprosin-1 cDNA sequence, GenBank NM_025994.3) of swiprosin-1 (LV-SWI) were produced by the Shanghai Innovation Biotechnology Co. RAW264.7 cells were transfected with LV-SWI or control lentiviruses without the swiprosin-1 CDS sequence (LV-GFP) for at least 72 hours.

Purification of $T$ cells. Mice splenocytes were suspended in RPMI medium containing 10\% FBS and were then applied to a nylon fiber column (Polysciences) according to the manufacturer's instructions. Briefly, scrubbed nylon fibers were placed into a 30-ml syringe and were then autoclaved for sterility. The columns were equilibrated with $30 \mathrm{ml}$ of RPMI culture media, sealed, and incubated for 30 minutes at $37^{\circ} \mathrm{C}$ in $5 \% \mathrm{CO}_{2}$. The spleens were removed, ground through a $70-\mu \mathrm{m}$ cell strainer, washed with HBSS, and treated with RBC lysis buffer. A total of $1 \times 10^{8}$ cells were resuspended in $3 \mathrm{ml}$ of warm RPMI and loaded onto the column. The column was sealed and incubated at $37^{\circ} \mathrm{C}$ and $5 \% \mathrm{CO}_{2}$ for 1 hour. Then, the cells were eluted with $10 \mathrm{ml}$ of warm RPMI.

Preparation of the peritoneal macrophages. The peritoneal macrophages were obtained from the mice after i.p. injection of $3 \mathrm{ml}$ of $3 \%$ thioglycolate as described previously (42). Briefly, the mice were sacrificed, and the macrophages were isolated by lavage with $5 \mathrm{ml}$ of RPMI (Gibco), washed twice with PBS after 3 hours of adherence, cultured in RPMI at $37^{\circ} \mathrm{C}$ and $5 \% \mathrm{CO}_{2}$, and finally stimulated with $1 \mu \mathrm{g} / \mathrm{ml} \mathrm{LPS}$ to harvest the whole cell lysates and supernatants. The isolated cells were used for cytokine analysis, Western blotting, quantitative PCR (qPCR), wound healing assay, flow cytometry, and phagocytosis and killing assays.

Cytokine analysis. The IL- $1 \beta$, IL- 6 , TNF- $\alpha$, IL-10, and IFN- $\gamma$ concentrations were measured using mouse IL-1 $\beta$, IL-6, TNF- $\alpha$, IL-10, and IFN- $\gamma$ ELISA kits (eBioscience) according to the manufacturer's instructions. Briefly, the capture antibody for each cytokine was first incubated overnight and then incubated with the sample to detect the rate of antibody-sample binding and HRP conjugation with the antibody. The optical density (OD) was measured at $450 \mathrm{~nm}$. Finally, the cytokine concentrations were calculated using the standard curve obtained in each experiment.

Western blotting. The protein samples were electrophoresed in $8 \%-12 \%$ SDS-PAGE gels and transferred onto nitrocellulose (NC) membranes (Amersham). After being blocked with 5\% BSA in PBS for 1 hour at 
room temperature, they were probed using primary antibodies. The bands were visualized and quantified with the Odyssey Infrared Imaging System (LI-COR). All the immunoblotting experiments were repeated at least 3 times.

$q P C R$. Total RNA was isolated from the primary peritoneal macrophages using Trizol Reagent (Takara). Total RNA (500 ng) was reverse transcribed with a Prime Script RT reagent kit from Takara, and then cDNA was synthesized by the SYBR Green Assay according to the manufacturer's instructions. After reverse transcription, the cDNA was used as a template for PCR. qPCR was carried out on an ABI 7500 real-time PCR system (Applied Biosystems) using the following primers. $\beta$-Actin or GAPDH was used as the reference gene. $\beta$-Actin (mice) forward primer, 5'-GTCCCTCACCCTCCCAAAAG-3'; reverse primer, 5'-GCTGCCTCAACACCTCAACCC-3'. IL-1 $\beta$ (mice) forward primer, 5'-CTCACAAGCAGAGCACAAGC-3'; reverse primer, 5'-CAGTCCAGCCCATACTTTAGG-3'. IL-6 (mice) forward primer, 5'-CGGAGAGGAGACTTCACAGAG-3'; reverse primer, 5'-CATTTCCACGATTTCCCAGA-3'. TNF(mice) forward primer, CATTTCCACGATTTCCCAGA-3'; reverse primer, 5'-GGAAAGCCCATTTGAGTCCT-3'. IFN- $\gamma$ (mice) forward primer, 5'-GGTCAACAACCCACAGGTCC-3'; reverse primer, 5'-CGAATCAGCAGCGACTCCTT-3'. IL-10 (mice) forward primer, 5'-GCTATGTTGCCTGCTCTT-3'; reverse primer, 5'-ATGCTCCTTGATTTCTGG-3'. TLR4 (mice) forward primer, 5'-TCGCTCAATCTGTCTTTCAC-3'; reverse primer, 5'-TCTGACGATGTGGGTAGCG-3'. CD14 (mice) forward primer, 5'-AGACCTGAGTGGCAATGAGC-3'; reverse primer, 5'-TCATCTGGGCTAGGGTTC-3'. Swiprosin-1 (mice) forward primer, 5'-GTGTCCGTTGCTGTGTTGTG-3'; reverse primer, 5'-CCCCTCCGATTCTCATAGGT-3'. GAPDH (human) forward primer, 5'-ATGGTTTACATGTTCCAATATGATT-3'; reverse primer, 5'-AATCATATTGGAACATGTAAACCAT-3'. Swiprosin-1 (human) forward primer, 5'-ACCTCAACCAGGGCATCG-3'; reverse primer, 5'-TCAAAGTCCTCATCCACCTCC-3'.

Wound healing assay. Peritoneal macrophages isolated from WT and $\mathrm{KO}$ mice were seeded at a density of $1 \times 10^{6}$ cells per well in a 6-multiwell plate and were incubated at $37^{\circ} \mathrm{C} 5 \% \mathrm{CO}_{2}$ overnight. Then, a horizontal slit was made in the center of each well using a white tip. The cells were incubated in the absence or presence of LPS $(1 \mu \mathrm{g} / \mathrm{ml})$. Cell migration into the slit of the confluent well was assessed at $0,3,6,12,24$, and 48 hours.

Flow cytometry. The cells isolated from the peritoneal fluid, and spleens of the mice after LPS or PBS treatment were incubated with F4/80-PE (eBioscience) and CD11b-FITC (BioLegend) fluorescent antibodies for 30 minutes and were washed twice with staining buffer before measurement with a BD FACSCalibur flow cytometer (BD Biosciences).

Phagocytosis and killing assay. A total of $1 \times 10^{7}$ macrophages and $1 \times 10^{9} \mathrm{BL}-21$ bacteria were cocultured as described previously (43). Briefly, the culture tube was incubated at $37^{\circ} \mathrm{C}$ for 2 hours and was centrifuged at $250 \mathrm{~g}$ for 5 minutes. The supernatant was diluted $1 \times 10^{5}$ times, and the pellets were resuspended in $0.1 \%$ Triton-X100 solution and diluted $1 \times 10^{4}$ times, from which $100 \mu 1$ of the dilution was plated onto an LB-agar plate and was incubated for 24 hours. Cell colonies were counted using the following equations:

Number of phagocytotic cells $=$ number of colonies in the control supernatant - number of colonies in the experimental supernatant

Killing number $=$ phagocytosis number - colony number of pellets

Statistics. The data are expressed as the mean \pm SEM. Statistical comparisons were performed using 2-tailed Student's $t$ test or 1-way ANOVA. SPSS 17.0 (SPSS Inc.) was used for the statistical analyses. $P<0.05$ was considered statistically significant.

Study approval. All animal experiments were carried out in adherence with the NIH Guide for the Care and Use of Laboratory Animals (National Academies Press, 2011) and were approved by the Second Military Medical University Committee on Animal Care (EC11-055).

\section{Author contributions}

SZ contributed acquisition of data, analysis and interpretation of data, methodology, and writing the original draft; YT contributed conceptualization, acquisition of data, project administration, and unpublished essential data; YMS, YL, and RMW contributed acquisition of data, methodology, and unpublished essential data; LL and LCZ contributed funding acquisition, project administration, writing the review, and editing; YBC contributed methodology and editing; and ZBW contributed conceptualization, resources, supervision, funding acquisition, writing the original draft, project administration, writing the review, and editing. 


\section{Acknowledgments}

We thank Tong-Hui Ma (Dalian Medical University, China) and Wei-Heng Su (Jilin University, China) for constructing the swiprosin-1-KO mouse. We thank Chun-Yan Xia (Shanghai Changzheng Hospital, China) for helping to analyze the pathological sections. We thank Pei Wang and Chong Liu (Second Military Medical University, China) for his comments on the writing of the article. This study was supported by grants from the National Natural Science Foundation of China (No 81402941 and 81473258), the Shanghai Municipal Commission of Health and Family Planning (No 20144Y0204, 20164Y0051 and 201540294) and the Science and Technology Commission of Shanghai Municipality (No 15140904600 and 16ZR1434400).

Address correspondence to: Zhi-Bin Wang, College of Pharmacy, Second Military Medical University, Guohe Road 325, Shanghai, 200433, China. Phone: 86.21.81871213; Email: methyl@smmu.edu.cn. Or to: Li-Chao Zhang, Department of Pharmacy, Shanghai Municipal Hospital of Traditional Chinese Medicine, 274 Zhi Jiang Middle Road, Shanghai, 200071, China. Phone: 86.15618776097; Email: changhaiskin@163. com. Or to: Ling Li, Department of Pharmacology, College of Pharmacy, The Second Military Medical University, 325 Guohe Road, Shanghai 200433, China. Phone:86.13601838793; Email: Lingli_z163@163.com.

1. Shankar-Hari M, et al. Developing a New Definition and Assessing New Clinical Criteria for Septic Shock: For the Third International Consensus Definitions for Sepsis and Septic Shock (Sepsis-3). JAMA. 2016;315(8):775-787.

2. Deutschman CS, Tracey KJ. Sepsis: current dogma and new perspectives. Immunity. 2014;40(4):463-475.

3. Cavaillon JM, Eisen D, Annane D. Is boosting the immune system in sepsis appropriate? Crit Care. 2014;18(2):216.

4. Patil NK, Bohannon JK, Sherwood ER. Immunotherapy: A promising approach to reverse sepsis-induced immunosuppression. Pharmacol Res. 2016;111:688-702.

5. Martin GS, Mannino DM, Moss M. The effect of age on the development and outcome of adult sepsis. Crit Care Med. 2006;34(1):15-21.

6. Boomer JS, et al. Immunosuppression in patients who die of sepsis and multiple organ failure. JAMA. 2011;306(23):2594-2605

7. Ayala A, Chaudry IH. Immune dysfunction in murine polymicrobial sepsis: mediators, macrophages, lymphocytes and apoptosis. Shock. 1996;6 Suppl 1:S27-S38.

8. Dalton DK, Pitts-Meek S, Keshav S, Figari IS, Bradley A, Stewart TA. Multiple defects of immune cell function in mice with disrupted interferon-gamma genes. Science. 1993;259(5102):1739-1742.

9. Traeger $\mathrm{T}$, et al. Selective depletion of alveolar macrophages in polymicrobial sepsis increases lung injury, bacterial load and mortality but does not affect cytokine release. Respiration. 2009;77(2):203-213.

10. Cohen HB, Briggs KT, Marino JP, Ravid K, Robson SC, Mosser DM. TLR stimulation initiates a CD39-based autoregulatory mechanism that limits macrophage inflammatory responses. Blood. 2013;122(11):1935-1945.

11. Belikoff BG, et al. A2B adenosine receptor blockade enhances macrophage-mediated bacterial phagocytosis and improves polymicrobial sepsis survival in mice. J Immunol. 2011;186(4):2444-2453.

12. Huang X, et al. PD-1 expression by macrophages plays a pathologic role in altering microbial clearance and the innate inflammatory response to sepsis. Proc Natl Acad Sci USA. 2009;106(15):6303-6308.

13. Vuadens F, Rufer N, Kress A, Corthésy P, Schneider P, Tissot JD. Identification of swiprosin 1 in human lymphocytes. Proteomics. 2004;4(8):2216-2220.

14. Mielenz D, et al. Lipid rafts associate with intracellular B cell receptors and exhibit a B cell stage-specific protein composition. J Immunol. 2005;174(6):3508-3517.

15. Avramidou A, Kroczek C, Lang C, Schuh W, Jäck HM, Mielenz D. The novel adaptor protein Swiprosin-1 enhances BCR signals and contributes to BCR-induced apoptosis. Cell Death Differ. 2007;14(11):1936-1947.

16. Vega IE, et al. A novel calcium-binding protein is associated with tau proteins in tauopathy. J Neurochem. 2008;106(1):96-106.

17. Ferrer-Acosta Y, et al. EFhd2 is a novel amyloid protein associated with pathological tau in Alzheimer's disease. $J$ Neurochem. 2013;125(6):921-931.

18. Thylur RP, et al. Swiprosin-1 is expressed in mast cells and up-regulated through the protein kinase C beta I/eta pathway. J Cell Biochem. 2009;108(3):705-715

19. Meador BM, Krzyszton CP, Johnson RW, Huey KA. Effects of IL-10 and age on IL-6, IL-1beta, and TNF-alpha responses in mouse skeletal and cardiac muscle to an acute inflammatory insult. J Appl Physiol. 2008;104(4):991-997.

20. Wright F, et al. Inflammatory pathway genes associated with inter-individual variability in the trajectories of morning and evening fatigue in patients receiving chemotherapy. Cytokine. 2017;91:187-210.

21. Pangault C, Le Tulzo Y, Tattevin P, Guilloux V, Bescher N, Drénou B. Down-modulation of granulocyte macrophage-colony stimulating factor receptor on monocytes during human septic shock. Crit Care Med. 2006;34(4):1193-1201.

22. Matute-Bello G, Frevert CW, Martin TR. Animal models of acute lung injury. Am J Physiol Lung Cell Mol Physiol. 2008;295(3):L379-L399.

23. Liu W, Wu H, Chen L, Wen Y, Kong X, Gao WQ. Park7 interacts with p47(phox) to direct NADPH oxidase-dependent ROS production and protect against sepsis. Cell Res. 2015;25(6):691-706.

24. Alazawi W, et al. Stat2 loss leads to cytokine-independent, cell-mediated lethality in LPS-induced sepsis. Proc Natl Acad Sci USA. 2013;110(21):8656-8661.

25. Steen HC, Gamero AM. STAT2 phosphorylation and signaling. JAKSTAT. 2013;2(4):e25790.

26. Kwon MS, et al. Swiprosin-1 is a novel actin bundling protein that regulates cell spreading and migration. $P L O S O N E$. 
2013;8(8):e71626.

27. Park KR, et al. Structural implications of Ca2+-dependent actin-bundling function of human EFhd2/Swiprosin-1. Sci Rep . 2016;6:39095

28. Park KR, et al. Structural mechanism underlying regulation of human EFhd2/Swiprosin-1 actin-bundling activity by Ser183 phosphorylation. Biochem Biophys Res Commun. 2017;483(1):442-448.

29. Munoz C, Carlet J, Fitting C, Misset B, Blériot JP, Cavaillon JM. Dysregulation of in vitro cytokine production by monocytes during sepsis. J Clin Invest. 1991;88(5):1747-1754

30. Prucha M, Zazula R, Russwurm S. Immunotherapy of Sepsis: Blind Alley or Call for Personalized Assessment? Arch Immunol Ther Exp (Warsz). 2017;65(1):37-49.

31. Arens C, et al. Sepsis-induced long-term immune paralysis--results of a descriptive, explorative study. Crit Care. 2016;20:93.

32. Lukaszewicz AC, et al. Monocytic HLA-DR expression in intensive care patients: interest for prognosis and secondary infection prediction. Crit Care Med. 2009;37(10):2746-2752.

33. Astiz M, Saha D, Lustbader D, Lin R, Rackow E. Monocyte response to bacterial toxins, expression of cell surface receptors, and release of anti-inflammatory cytokines during sepsis. J Lab Clin Med. 1996;128(6):594-600.

34. Venet F, Lukaszewicz AC, Payen D, Hotchkiss R, Monneret G. Monitoring the immune response in sepsis: a rational approach to administration of immunoadjuvant therapies. Curr Opin Immunol. 2013;25(4):477-483.

35. Hotchkiss RS, Monneret G, Payen D. Sepsis-induced immunosuppression: from cellular dysfunctions to immunotherapy. Nat Rev Immunol. 2013;13(12):862-874.

36. Döcke WD, et al. Monocyte deactivation in septic patients: restoration by IFN-gamma treatment. Nat Med. 1997;3(6):678-681.

37. Kumar CS, Mariano TM, Noe M, Deshpande AK, Rose PM, Pestka S. Expression of the murine interferon gamma receptor in Xenopus laevis oocytes. J Biol Chem. 1988;263(27):13493-13496.

38. Sen S, Roy K, Mukherjee S, Mukhopadhyay R, Roy S. Restoration of IFN $\gamma$ R subunit assembly, IFN $\gamma$ signaling and parasite clearance in Leishmania donovani infected macrophages: role of membrane cholesterol. PLoS Pathog. 2011;7(9):e1002229.

39. Kroczek C, et al. Swiprosin-1/EFhd2 controls B cell receptor signaling through the assembly of the B cell receptor, Syk, and phospholipase C gamma2 in membrane rafts. J Immunol. 2010;184(7):3665-3676.

40. Wang ZB, et al. Low level of swiprosin-1/EFhd2 in vestibular nuclei of spontaneously hypersensitive motion sickness mice. Sci Rep. 2017;7:40986.

41. Rittirsch D, Huber-Lang MS, Flierl MA, Ward PA. Immunodesign of experimental sepsis by cecal ligation and puncture. Nat Protoc. 2009;4(1):31-36.

42. Li M, Lin F, Lin Y, Peng W. Extracellular polysaccharide from Bordetella species reduces high glucose-induced macrophage apoptosis via regulating interaction between caveolin-1 and TLR4. Biochem Biophys Res Commun. 2015;466(4):748-754.

43. Mombelli M, et al. Histone deacetylase inhibitors impair antibacterial defenses of macrophages. J Infect Dis. 2011;204(9):1367-1374. 